\title{
Performance of Adaptive Multiuser Detectors in Space-Time Coded CDMA Systems
}

\author{
M. M. Alam and A. Chockalingam \\ Department of Electrical Communication Engineering, Indian Institute of Science, Bangalore 560012. INDIA
}

\begin{abstract}
In this paper, we present a comparative performance evaluation of adaptive multiuser detectors, including stochastic gradient (SG) and recursive least squares (RLS) algorithms (which require training data), and minimum output energy (MOE) and subspace-based MMSE (S-MMSE) algorithms (which do not require training data), under near-far conditions in a space-time coded CDMA system. We show that, in a near-far multituser scenario, increasing the number of transmit antennas degrade the near-far resistance performance of the SG and MOE detectors significantly at high near-far ratios, to the extent that the diversity benefit of multiple transmit antennas is nullified. The RLS and S-MMSE algorithms, on the other hand, are shown to maintain their near-far resistance without loosing much on the diversity gain, when the number of transmit antennas is increased.
\end{abstract}

Keywords: Space-time codes. CDMA. adaptive multiuser detection.

\section{INTRODUCTION}

It has been shown that space-time coded transmission using multiple transmit antennas can offer the benefits of transmit diversity and high data rate transmission on wireless fading channels [1],[2], which has generated lot of interest in using this technique in communication systems design (e.g., in 3G). Also, multiuser detection schemes for interference rejection, which can significantly enhance the receiver performance and increase the capacity of code division multiple access (CDMA) systems, have been extensively studied the in literature, mainly for single transmit antenna systems [3]. Investigation of multiuser detection algorithms and their performances in space-time coded systems with multiple transmit antennas has been gaining importance [4],[5].

Adaptive detectors are of interest since non-adaptive detectors require estimation of various parameters of all the users and involve matrix inversion which becomes computationally intensive as the number of users in the system (and the number of transmit antennas) increases. Our interest here is to develop adaptive receiver structures for space-time coded CDMA and evaluate their performances. We first derive a discrete-time vector model for the received signal using orthonormal projections in a space-time coded CDMA system, wherein we assume that only the timing of the desired user is known at the receiver. Using the vector model,

This work was supported in part by the Swarnajayanti Fellowship. Depanment of Science and Technology. New Delhi. India. we then develop the structure of various adaptive multiuser detectors with only the knowledge of the channel impulse response and the signature waveform of the desired user being available in the detection process. The various adaptive algorithms considered include the stochastic gradient (SG) and recursive least squares (RLS) algorithms (which require training data), and minimum output energy (MOE) and subspace-based MMSE (S-MMSE) algorithms (which do not require training data). We evaluate and compare the nearfar resistance performance of the above detectors. We also evaluate the convergence behaviour of the detectors in a dynamic near-far scenario, where users can randomly enter into and exit form the system. Our performance results show that the RLS and subspace-based MMSE methods offer better near-far resistance and convergence behaviour compared to the SG and MOE algorithms, when the number of transmit antennas is increased.

\section{SYSTEM MODEL}

We consider a $K$-user asynchronous CDMA system with $M$ transmit antennas per user. Users transmit frames of data blocks with $Q$ bits per data block. Let $b_{i q}(m), i \in$ $\{1,2, \ldots, K\}, q \in\{1,2, \ldots, Q\}$, be the $q^{\text {th }}$ bit of the $m^{\text {th }}$ block of the $i^{t h}$ user, transmitted in a time interval of length $T$. The bits in a data block are mapped on to the $M$ transmit antennas using orthogonal space-time block codes (STBC). We assume that the channel fading is quasi-static and the quasi-static interval is $Q T$ time units, where $Q=2^{r}, r$ being the smallest integer satisfying $Q \geq M$ [2]. For square real orthogonal STBC with $M=Q=\overline{8}$, the transmission matrix $\mathrm{B}$ is [1]

$\mathbf{B}=\left[\begin{array}{cccccccc}b_{1} & b_{2} & b_{3} & b_{4} & b_{5} & b_{6} & b_{7} & b_{8} \\ b_{2} & -b_{1} & -b_{4} & b_{3} & -b_{6} & b_{5} & b_{8} & -b_{7} \\ b_{3} & b_{4} & -b_{1} & -b_{2} & -b_{7} & -b_{8} & b_{5} & b_{6} \\ b_{4} & -b_{3} & b_{2} & -b_{1} & -b_{8} & b_{7} & -b_{6} & b_{5} \\ b_{5} & b_{6} & b_{7} & b_{8} & -b_{1} & -b_{2} & -b_{3} & -b_{4} \\ b_{6} & -b_{5} & b_{8} & -b_{7} & b_{2} & -b_{1} & b_{4} & -b_{3} \\ b_{7} & -b_{8} & -b_{5} & b_{6} & b_{3} & -b_{4} & -b_{1} & b_{2} \\ b_{8} & b_{7} & -b_{6} & -b_{5} & b_{4} & b_{3} & -b_{2} & b_{1}\end{array}\right]$.

In the above transmission matrix, the columns represent the transmit antenna index and the rows represent the bit interval index. The transmission matrix $\mathbf{B}$ for other real orthogonal designs for $M, Q<8$ can be obtained to be the upper leftmost submatrix of $\mathbf{B}$ of $\operatorname{order} Q \times M$. For illustration, 
we use $M \quad Q \quad$ for the derivation of the vector model. Extending the model for other values of $M, Q \leq 8$ is straightforward.

For the $M=Q=2$ system, the received signal on a receive antenna can be written using (1) as

$$
\begin{aligned}
& y(t)=y_{1}(t)+y_{2}(t)+z(t), \\
& y_{1}(t)=\sum_{m=-L}^{L} \sum_{i=1}^{K} A_{i 1} h_{i 1}(m)\left\{b_{i 1}(m) s_{i 1}^{(m)}+b_{i 2}(m) s_{i 2}^{(m)}\right\}, \\
& y_{2}(t)=\sum_{m=-L}^{L} \sum_{i=1}^{K} A_{i 2} h_{i 2}(m)\left\{b_{i 2}(m) s_{i 1}^{(m)}-b_{i 1}(m) s_{i 2}^{(m)}\right\} .
\end{aligned}
$$

In the above, $y_{p}(t), p \in\{1,2\}$ is the signal component due to the $p^{t / h}$ transmit antenna, $A_{i p}$ is the transmit amplitude on the $p^{\text {th }}$ transmit antenna of the $i^{\text {th }}$ user, $h_{\text {ip }}(m)$ is the complex channel gain for the $m^{t h}$ block from the $p^{t h}$ transmit antenna of the $i^{\text {th }}$ user, and $s_{i q}^{(m)}$ represents the signature waveform of the $i^{\text {th }}$ user for the $q^{\text {th }}$ bit in a block, $q \in\{1,2\}$, given by

$$
s_{i q}^{(m)}=s_{i}\left(t-m Q T-\overline{q-1} T-\tau_{i}\right),
$$

where $s_{i}(t)$ is a unit energy signature waveform time limited in the interval $\{0, T]$, and $\tau_{i}$ is the random time delay of the $i^{t / k}$ user, which we assume to be independent of the transmitting antenna and to satisfy $\tau_{j} \geq \tau_{i}$ for $j>i$. Also, $z(t)$ is a zero mean complex Gaussian noise process with variance $2 \sigma^{2}$.

Let $\left\{\phi_{1}, \phi_{2}, \ldots, \phi_{D}\right\}$ be a set of $D$ orthonormal signals defined on $[0, T]$. Also define

$$
\phi_{j}^{(q)}(t)=\phi_{j}(t-\overline{q-1} T), q=1, \ldots, Q, j=1, \ldots, D .
$$

Since the signature waveforms of some of the interferers may not be known a priori, we project the received signal in the $n^{\text {th }}$ block in (3) on to the $D$-dimensional space as follows:

$$
y_{j}^{\{(n)}(n)=\int_{n Q T+r}^{(n+1) Q T+\tau} y(t) \phi_{j}^{q}(t-n Q T-\tau) d t, q=1, \ldots, Q, j=1, \ldots, D,(7)
$$

where $\tau$ is the timing of the desired user. We define the following projections of the $k^{t h}$ user's signature waveform on to the $j^{\text {th }}$ component of the orthonormal basis as

$s_{k j}(\delta)=\int_{0}^{T-\delta} s_{k^{\prime}}(t+\delta) \phi_{j}(t) d t, s_{k j}(\delta)=\int_{T-\delta}^{T} s_{k}(t-T+\delta) \phi_{j}(t) d t, \quad{ }^{\prime} x_{j}$

where $\delta \in[0, T]$. Let the $k^{\text {th }}$ user be the desired user (i.e., $\left.\tau_{k}=\tau\right)$ and $\delta_{i}=\left|\tau_{i}-\tau\right|, i \in\{1,2, \ldots, K\}$. Using (8), we define the following matrices of order $D \times K$ :

$$
\begin{gathered}
\mathbf{S}_{\mathbf{0}}=\left[\begin{array}{ccccc}
s_{12}\left(\delta_{1}\right) & \ldots & s_{k_{1}}(0) & \ldots & \bar{s}_{K 1}\left(T-\delta_{K}\right) \\
s_{12}\left(\delta_{1}\right) & \ldots & s_{k 2}(0) & \ldots & \bar{s}_{K 2}\left(T-\delta_{K}\right) \\
\vdots & \vdots & \vdots & \vdots & \vdots \\
s_{1 D}\left(\delta_{1}\right) & \ldots & s_{k+D}(0) & \ldots & \bar{s}_{K D}\left(T-\delta_{K}\right)
\end{array}\right], \\
\mathbf{S}_{-1}=\left[\begin{array}{cccccc}
0 & \ldots & 0 & s_{(k+1) 1}\left(T-\delta_{k+1}\right) & \ldots & s_{K 1}\left(T-\delta_{K}\right) \\
0 & \ldots & 0 & s_{(k+1) 2}\left(T-\delta_{k+1}\right) & \ldots & s_{K 2}\left(T-\delta_{K}\right) \\
\vdots & \vdots & \vdots & \vdots & \vdots & \vdots \\
0 & \ldots & 0 & s_{(k+1) D}\left(T-\delta_{k+1}\right) & \ldots & s_{K D}\left(T-\delta_{K}\right)
\end{array}\right],
\end{gathered}
$$




$$
\begin{aligned}
\mathbf{H}(n) & =\left[\mathbf{h}_{1}^{H}(n), \mathbf{h}_{2}^{H}(n), \ldots, \mathbf{h}_{Q K}^{H}(n)\right]^{H} \\
\mathbf{X}_{j} & =\left[\mathbf{s}_{1}^{(j)}, \mathbf{s}_{2}^{(j)}, \ldots, \mathbf{s}_{Q K}^{(j)}\right],
\end{aligned}
$$

where $\mathbf{h}_{i}(n)$ is the $i^{t h}$ row of $\mathbf{H}(n), \mathbf{s}_{i}^{(j)}$ is the $i^{\text {th }}$ column of $\mathrm{X}^{(j)}$. Because of the orthogonal nature of the space-time block codes used [2], we can show that

$$
\operatorname{Re}\left[\mathbf{h}_{i}(n) \mathbf{h}_{j}^{H}(n)\right]=\delta_{i j}\left\|\mathbf{h}_{i}(n)\right\|^{2},
$$

where $\delta_{i j}$ is the Kronecker Delta function. If we extract those rows from $\mathbf{H}(n)$ which contains the complex channel gains of the desired user, i.e., the $k^{\text {th }}$ user, we can define a reduced channel matrix for the $k^{t h}$ user, as

$$
\mathbf{G}_{k}^{H}(n)=\left[\mathbf{h}_{k}^{H}(n), \mathbf{h}_{K+k}(n), \ldots, \mathbf{h}_{(Q-1) K+k}^{H}(n)\right]^{H} .
$$

which satisfies the following property:

$$
\begin{gathered}
\operatorname{Re}\left[\mathbf{G}_{k}^{H}(n) \mathbf{G}_{k}(n)\right]=\operatorname{diag}\left[\mathbf{P}_{1}(n), \ldots, \mathbf{P}_{Q}(n)\right], \\
\mathbf{P}_{2}(n)=\ldots=\mathbf{P}_{Q}(n)=\operatorname{diag}\left[0, \ldots, \sum_{p=1}^{M} A_{k_{p}}^{2}\left|h_{k_{p}}(n)\right|^{2}, \ldots, 0\right]_{K \times K} .
\end{gathered}
$$

Using (23), we can finally rewrite (18) as

$$
\begin{array}{r}
\mathbf{y}(n)=\sum_{i=1}^{Q K}\left[\mathbf{s}_{i}^{(-1)} \mathbf{h}_{i}(n-1) \mathbf{b}(n-1)+\mathbf{s}_{i}^{(0)} \mathbf{h}_{i}(n) \mathbf{b}(n)\right. \\
\left.+\mathbf{s}_{i}^{(1)} \mathbf{h}_{i}(n+1) \mathbf{b}(n+1)\right]+\mathbf{z}(n) .
\end{array}
$$

In the derivations of the various adaptive multiuser detectors, we assume perfect knowledge of the $k^{t h}$ user's (i.e., desired user's) reduced channel matrices, $\mathbf{G}_{k}(n)$, and signature vectors, $\mathbf{s}_{(q-1) K+k^{3}}^{(0)}$ (or training bits, $\left.b_{k q}(n)\right), q \in\{1,2, \ldots, Q\}$. An estimate of the desired user's $Q$ bits is given by

$$
\dot{b}_{k q}(n)=\operatorname{sgn}\left[\operatorname{Re}\left(\mathbf{G}_{k}^{H}(n) \mathbf{M}_{k}^{T}(n) \mathbf{y}(n)\right)_{(q-1) K+k}\right] \text {, }
$$

where $q \in\{1,2, \ldots, Q\}$ and $\mathbf{M}_{k}^{T}(n)$ is a linear transformation which is applied to the received signal vector at the $n^{\text {th }}$ block.

The conventional MF detector correlates the input signal vector $\mathrm{y}(n)$, with the signature vectors $\mathrm{s}_{(q-1) K+k}^{(0)}, q \in$ $\{1,2, \ldots, Q\}$ and the transformation $\mathbf{M}_{k}^{T}(n)$ in (30) is given by $\mathbf{M}_{k}(n)=\left[\mathbf{s}_{k}^{(0)}, \mathbf{s}_{K+k}^{(0)}, \ldots, \mathbf{s}_{(Q-1) K+k}^{(0)}\right]$.

\section{A. Stochastic gradient (SG) algorithm}

In the SG algorithm, we adaptively minimize

$$
\begin{array}{r}
M S E_{i}=\xi_{i}=E\left[\left|\mathbf{h}_{i}(n) \mathbf{b}(n)-\mathbf{m}_{i}^{T}(n) \mathbf{y}(n)\right|^{2}\right], \\
i=\overline{q-1} K+k, q=1, \ldots, Q,
\end{array}
$$

where $\mathbf{m}_{i}(n)$ are the $Q$ real weight vectors at the $n^{\text {th }}$ block for the $k^{t h}$ user. The adaptive algorithm is given by

$$
\mathbf{m}_{i}(n)=\mathbf{m}_{i}(n-1)-\mu \nabla \xi_{i},
$$

where $\mu$ is the step size. An estimate of $\nabla \xi_{i}$ is given by [3]

$$
\begin{aligned}
\hat{\nabla} \xi_{i} & =\nabla_{\mathbf{m}_{i}(n)}\left[\left|\mathbf{h}_{i}(n) \mathbf{b}(n)-\mathbf{m}_{i}^{T}(n) \mathbf{y}(n)\right|^{2}\right] \\
& =\operatorname{Re}\left[\mathbf{y}(n) \mathbf{y}^{H}(n) \mathbf{m}_{i}(n)-\mathbf{y}(n) \mathbf{b}^{T}(n) \mathbf{h}_{i}(n)\right] .
\end{aligned}
$$

Substituting the estimate from (33) into (32), we get $\mathbf{m}_{i}(n)=\mathbf{m}_{i}(n-1)+\mu R e\left[\mathbf{y}(n) \mathbf{b}^{T}(n) \mathbf{h}_{i}(n)-\mathbf{y}(n) \mathbf{y}^{n}(n) \mathbf{m}_{i}(n-1)\right]$. (34) It is noted that we need training bits of the desired user, $b_{k q}(n), q \in\{1,2, \ldots, Q\}$, via $\mathbf{b}(n)$ for the adaptation process. The linear transformation in (30) can be written as

$$
\mathbf{M}_{k}(n)=\left[\mathbf{m}_{k}(n), \mathbf{m}_{K+k}(n), \ldots, \mathbf{m}_{(Q-1) K+k}(n)\right] .
$$

\section{B. Recursive least squares (RLS) algorithm}

The optimum weight vectors $\mathrm{m}_{i}, i=(q-1) K+k, q \in$ $\{1,2, \ldots, Q\}$ which minimizes (31) is given by the solution of

$$
\operatorname{Re}\left[E\left[\mathbf{y}(n) \mathbf{y}^{H}(n)\right]\right] \mathbf{m}_{i}^{*}=\operatorname{Re}\left[E\left[\mathbf{y}(n) \mathbf{b}^{T}(n) \mathbf{h}_{i}^{H}(n)\right]\right] .
$$

Replacing expectation in (36) by the time average,

(27)

$\operatorname{Re}\left(\sum_{j=1}^{n} y(n) y^{H}(n)\right) m_{i}(n)=R e\left(\sum_{j=1}^{n} y(n) b^{T}(n) h_{i}^{H}(n)\right)$.

Defining $\mathbf{Y}(n)=[\mathbf{y}(1), \mathbf{y}(2), \ldots, \mathbf{y}(n)], \mathbf{d}_{i}(n)=$

$\left[\mathbf{h}_{i}(1) \mathbf{b}(1), \mathbf{h}_{i}(2) \mathbf{b}(2), \ldots, \mathbf{h}_{i}(n) \mathbf{b}(n)\right],(37)$ can be written as

$\mathbf{R}(n) \mathbf{m}_{\mathbf{i}}(n)=\mathbf{r}_{i}(n)$,

$\mathbf{R}(n)=\operatorname{Re}\left[\mathbf{Y}(n) \mathbf{Y}^{H}(n)\right], \mathbf{r}_{i}(n)=\operatorname{Re}\left[\mathbf{Y}(n) \mathbf{d}_{i}^{H}(n)\right]$.

Now,

$$
\begin{gathered}
\mathbf{R}(n+1)=\mathbf{R}(n)+\operatorname{Re}\left[\mathbf{y}(n+1) \mathbf{y}^{H}(n+1)\right], \\
\mathbf{r}_{i}(n+1)=\mathbf{r}_{i}(n)+\operatorname{Re}\left[\mathbf{y}(n+1) \mathbf{b}^{T}(n+1) \mathbf{h}_{i}^{H}(n+1)\right] .
\end{gathered}
$$

Applying (38) for the $(n+1)^{\text {th }}$ block and using (40) and (41), it can be shown that

$\mathbf{m}_{i}(n+1)=\mathbf{m}_{i}(n)+\mathbf{R}^{-1}(n+1) R e\left[\mathbf{y}(n+1) \mathbf{b}^{T}(n+1) \mathbf{h}_{i}^{H}(n+1)\right.$

$$
\left.-\mathbf{y}(n+1) \mathbf{y}^{H}(n+1) \mathbf{m}_{i}(n)\right] \text {. }
$$

If we define

$$
\mathbf{P}(n+1)=[\operatorname{Re}(\mathbf{y}(n+1)), \operatorname{Im}(\mathbf{y}(n+1))],
$$

$\mathbf{p}_{i}(n+1)=\left[\operatorname{Re}\left(\mathbf{h}_{i}(n+1) \mathbf{b}(n+1)\right), \operatorname{Im}\left(\mathbf{h}_{i}(n+1) \mathbf{b}(n+1)\right)\right]$,

$i=\{g-1) K+k, g \in\{1,2, \ldots, Q\}$, we rewrite (42) as

$\mathbf{m}_{i}(n+1)=\mathbf{m}_{i}(n)+\mathbf{R}^{-1}(n+1) \mathbf{P}(n+1)\left[\mathbf{P}_{\mathbf{T}}^{T^{T}}(n+1)-\mathbf{P}^{T}(n+1) \mathbf{m}_{i}(n)\right]$.

The computation of $\mathbf{R}^{-1}(n+1)$ at each block is avoided by using the matrix inversion lemma to update the value of $\mathbf{R}^{-1}(n)$ to get $\mathbf{R}^{-1}(n+1)$. For nonsingular matrices $\mathbf{B}, \mathbf{D}$

$$
\begin{gathered}
\mathbf{A}=\mathbf{B}+\mathbf{C D C}^{T}, \\
\Rightarrow \mathbf{A}^{-1}=\mathbf{B}^{-1}-\mathbf{B}^{-1} \mathbf{C}\left(\mathbf{C}^{T} \mathbf{B}^{-1} \mathbf{C}+\mathbf{D}^{-1}\right)^{-1} \mathbf{C}^{T} \mathbf{B}^{-1} .
\end{gathered}
$$

Rewriting (40) using (43), $\mathbf{R}(n+1)=\mathbf{R}(n)+\mathbf{P}(n+1) \mathbf{P}^{T}(n+1)$, and setting $\mathbf{A}=\mathbf{R}(n+1), \mathbf{B}=\mathbf{R}(n), \mathbf{C}=\mathbf{P}(n+1), \mathbf{D}=\mathbf{I}_{2}$, we get

$$
\begin{aligned}
& \mathbf{R}^{-1}(n+1)=\mathbf{R}^{-1}(n)-\mathbf{R}^{-1}(n) \mathbf{P}(n+1) \\
& \quad \cdot\left(\mathbf{P}^{T}(n+1) \mathbf{R}^{-1}(n) \mathbf{P}(n+1)+\mathbf{I}_{2}\right)^{-1} \mathbf{P}^{T}(n+1) \mathbf{R}^{-1}(n) .
\end{aligned}
$$

It is noted that, now we only have to invert a $2 \times 2$ matrix at each iteration. But, the $Q D \times Q D$ matrix $\mathbf{R}(n)$ has $\operatorname{Rank}(\mathbf{R}(n))=\min \{n, Q D\}$, so the first $Q D$ observations can be used to form an initial estimate of $\mathbf{R}^{-1}(n)$ and hence $\mathbf{m}_{i}, i=(q-1) K+k, q \in\{1,2, \ldots, Q\}$, whereafter the recursive update algorithm given by $(45),(47)$ can be used. 


\section{Minimum output energy (MOE) algorithm}

The MOE detector minimizes the mean output energy [3]

$$
\begin{array}{r}
M O E_{i}=\xi_{i}=E\left[\left|\left(\mathbf{s}_{i}^{(0)}+\mathbf{x}_{i}(n)\right)^{T} \mathbf{y}(n)\right|^{2}\right], \\
i=\widetilde{q-1} K+k, q \in\{1,2, \ldots, Q\},
\end{array}
$$

subject to $\mathbf{x}_{i}^{T}(n) \mathbf{s}_{i}^{(0)}=0, \mathbf{s}_{i}^{(0)} \mathbf{s}_{i}^{(0)}=1$, for some real weight vector $\mathrm{x}_{i}(n)$. If we replace $\mathrm{m}_{i}(n)$ in $(31)$ by $\mathbf{s}_{i}^{(0)}+\mathrm{x}_{i}(n)$ and rewrite it in terms of (48), we get

$$
M S E_{i}=M O E_{i}-E\left[\left\|\mathbf{h}_{i}(n)\right\|^{2}\right] \text {. }
$$

Thus, minimizing the MSE is equivalent to minimizing the mean output energy, and the adaptation rule is given by

$$
\mathbf{x}_{i}(n)=\mathbf{x}_{i}(n-1)-\mu\left(\nabla \xi_{i}-\mathbf{s}_{i}^{(0) T} \nabla \xi_{i} \mathbf{s}_{i}^{(0)}\right),
$$

where $\mu$ is the step size. An estimate of $\nabla \xi_{i}$ is given by $\hat{\mathrm{r}} \xi_{i}=\nabla_{\mathbf{x}_{i}(n)}\left[\left|\left(\mathbf{s}_{i}^{(0)}+\mathbf{x}_{i}(n)\right)^{T} \mathbf{y}(n)\right|^{2}\right]=R e\left[\mathbf{y}(n) \mathbf{y}^{H}(n)\right]\left[\mathbf{s}_{i}^{(0)}+\mathbf{x}_{i}(n)\right]$.

Substituting the estimate from (51) into (50), we get $\mathbf{x}_{i}(n)=\mathbf{x}_{i}(n-1)+\mu\left[\mathbf{s}_{i}^{(0)} \mathbf{s}_{i}^{(0) T}-\mathbf{I}\right] \operatorname{Re}\left[\mathbf{y}(n) \mathbf{y}^{H}(n)\right]\left[\mathbf{s}_{i}^{(0)}+\mathbf{x}_{i}(n)\right]$.

The adaptation rule does not require training bits but requires the knowledge of the signature vector of the desired user. The linear transformation in (30) can be written as

$$
\mathbf{M}_{k}(n)=\left[\mathbf{s}_{k}^{(0)}+\mathbf{x}_{k}(n), \ldots, \mathbf{s}_{(Q-1) K+k}^{(0)}+\mathbf{x}_{(Q-1) K+k}(n)\right] .
$$

\section{Subspace based MMSE (S-MMSE)}

The real part of the autocorrelation matrix of the received

$$
\begin{aligned}
& \text { signal is given by } \\
& \qquad \mathbf{R}=\operatorname{Re}\left[E\left[\mathbf{y}(n) \mathbf{y}^{\mathrm{H}}(n)\right]=\sum_{j=-1}^{1} \sum_{i=1}^{Q K} \delta_{i}^{(j)} \boldsymbol{s}_{i}^{(j) T} E \mid\right\} \mathbf{h}_{i}(n) \|^{2}+2 \sigma^{2} x_{1}
\end{aligned}
$$

which can be obtained by time averaging sample autocorrelation functions. By performing an eigendecomposition of the matrix $\mathbf{R}$, we get

$$
\mathbf{R}=\mathbf{U} \Lambda \mathbf{U}^{T}=\left[\begin{array}{ll}
\mathbf{U}_{s} & \mathbf{U}_{n}
\end{array}\right]\left[\begin{array}{cc}
\Lambda_{s} & \mathbf{0} \\
\mathbf{0} & \Lambda_{n}
\end{array}\right]\left[\begin{array}{c}
\mathbf{U}_{s}^{T} \\
\mathbf{U}_{n}^{T}
\end{array}\right],
$$

where $\mathbf{U}=\left[\mathbf{U}_{s}, \mathbf{U}_{n}\right], \boldsymbol{\Lambda}=\operatorname{diag}\left(\boldsymbol{\Lambda}_{s}, \boldsymbol{\Lambda}_{n}\right) ; \boldsymbol{\Lambda}_{s}=$ $\operatorname{diag}\left(\lambda_{1}, \lambda_{2}, \ldots, \lambda_{Q K+K-1}\right)$ contains the $(Q K+K-1)$ largest eigenvalues of $\mathbf{R}$ and $\mathbf{U}_{s}=\left[\mathbf{u}_{1}, \ldots, \mathbf{u}_{Q K+K-1}\right]$ contains the corresponding orthonormal eigenvectors; $\boldsymbol{\Lambda}_{n}=$ $2 \sigma^{2} \mathbf{I}_{Q D-Q K-K+1}$ and $\mathbf{U}_{n}=\left\{\mathbf{u}_{Q K+K}, \ldots, \mathbf{u}_{Q D}\right\}$ contains the $(Q D-Q K-K+1)$ orthonormal eigenvectors corresponding to the eigenvalue $2 \sigma^{2}$. It is noted that the signature vectors $\mathrm{s}_{i}^{(0)}, i=\{q-1) K+k, q \in\{1,2, \ldots, Q\}$ lies in the range $\left(\mathbf{U}_{s}\right)$. The minimum mean square error detector can be derived by minimizing

$$
\xi\left(\mathbf{m}_{i}\right)=E\left[\left|\mathbf{h}_{i}(n) \mathbf{b}(n)-\mathbf{m}_{i}^{T} \mathbf{y}(n)\right|^{2}\right],
$$

subject to $\mathbf{m}_{i}^{T} \mathbf{s}_{i}^{(0)}=1$, where $\mathbf{m}_{i}, i=(q-1) K+k, q \epsilon$ $\{1,2, \ldots, Q\}$ are $Q$ real weight vectors for the $k^{\text {th }}$ user. By the method of Lagrange multipliers, we obtain [6]

$$
L\left(\mathbf{m}_{i}\right)=\xi\left(\mathbf{m}_{i}\right)-2 \mu\left(\mathbf{m}_{i}^{T} \mathbf{s}_{i}^{(0)}-1\right)
$$$$
\left.\left.\left.=\mathbf{m}_{i}^{T} \mathbf{R} \mathbf{m}_{i}-2 \mathbf{m}_{i}^{T} \mathbf{s}_{i}^{(0)} E \| \mathbf{h}_{i}(n)\right\}\right\}^{2}+E \mid\left\langle\mathbf{h}_{i}\right\}\right\}^{2}-2 \mu\left(\mathbf{m}_{i}^{T} \mathbf{s}_{i}^{(0)}-1\right) \text {. }
$$

Setting the gradient of $L\left(\mathbf{m}_{i}\right)$ with respect to $\mathbf{m}_{i}$ to zero and using the fact that $U_{n}^{T} \mathbf{s}_{i}^{(0)}=0$ and $\mathbf{m}_{i}^{T} \mathbf{s}_{i}^{(0)}=1$, the optimum
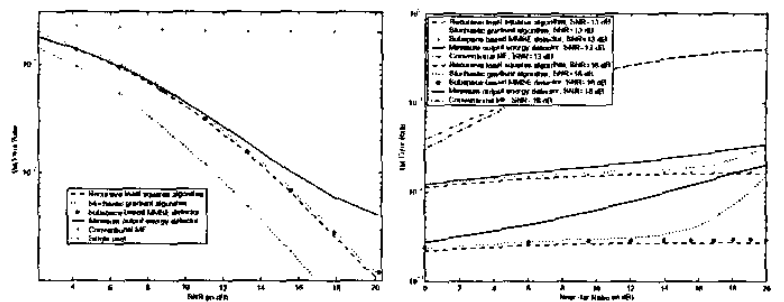

Fig. 1. BER performance of adaptive detectors for user 1 as a function of SNR and NFR. $M=2, \rho_{12}=-0.28 . \rho_{21}=-0.48$.

weight vectors can be written in terms of the signal subspace parameters $\left(\boldsymbol{\Lambda}_{s}, \mathbf{U}_{s}\right)$, as

$\mathbf{m}_{i}^{*}=\frac{\mathrm{U}_{s} \boldsymbol{\Lambda}_{s}^{-1} \mathbf{U}_{s}^{T} \mathbf{s}_{i}^{(0)}}{\mathbf{s}_{i}^{(0) T} \mathbf{U}_{s} \boldsymbol{\Lambda}_{s}^{-1} \mathbf{U}_{s}^{T} \mathbf{s}_{i}^{(0)}}, i=\overline{q-1} K+k, q \in\{1,2, \ldots, Q\}$.

\section{RESULTS AND DISCUSSION}

We evaluated the convergence and bit error performance of the adaptive algorithms discussed in section III, by using Gold codes to generate the signature vectors. In the left figure of Fig. 1, we compare the bit error rate (BER) performance of the various adaptive algorithms for a two user $(K=2)$ asynchronous CDMA system with two transmit antennas ( $M=2$ ) per user. The signature sequences of the two users are chosen such that the cross-correlations between them are given by $\rho_{12}=-0.28 . \rho_{21}=-0.48$. In the algorithms which require training data, we assume a training sequence of 1000 blocks. The near-far ratio ${ }^{1}$ (NFR) assumed is $9.54 \mathrm{~dB}$. The single user performance is also plotted for comparison. It is observed that the BER performance of the SG algorithm, the RLS algorithm and the S-MMSE algorithm are almost identical, whereas the MOE algorithm performs comparatively poor in the high SNR region. In the right figure of Fig. 1, we illustrate the BER performance of the various adaptive algorithms as a function of the near-far ratio. It is noted that for an SNR of $13 \mathrm{~dB}$, the BER performance of all the algorithms are almost independent of NFR, with marginal degradation of performance for the SG and MOE algorithms in the high NFR region (15-20 dB). However, for an SNR of $18 \mathrm{~dB}$, only the RLS algorithm and the S-MMSE algorithm are near-far resistant. The MOE algorithm performs the worst and shows a degradation (almost linear) with increase in the NFR. The SG algorithm retains the near-far resistance over a larger NFR range compared to MOE, but it also looses its near-far resistance in the high NFR region. This is because in the high NFR region, the noisy gradient estimate in (33) is no longer able to make the weight vectors in (34) roll down the performance surface in (31).

${ }^{1} \mathrm{NFR}$ is defined as $10 \log \frac{\sum_{p=1}^{M} \frac{A_{2 p}^{2}}{\sum_{p=1}^{M} A_{1_{p}}^{2}}}{{ }^{2}}$ assuming $E\left|h_{i q}(n)\right|^{2}=1$. 

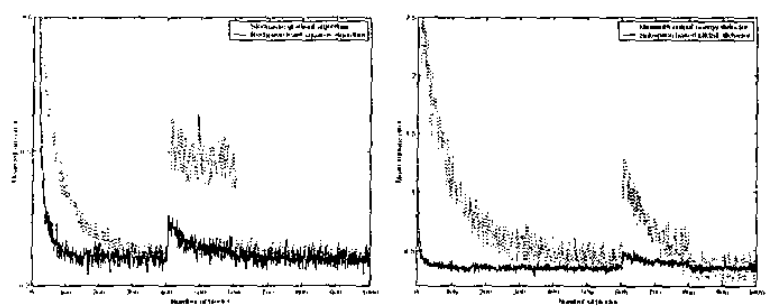

Fig. 2. Convergence plots of adaptive algoritlums. $\mathrm{SNR}=13,46 \mathrm{~dB}, M=$ $2, \rho_{12}=-0.28, \rho_{21}=-0.42$. NFR increased from 10 to $18 \mathrm{~dB}$ at $n=$ $400(600)$ and decreased to $10 \mathrm{~dB}$, at $n=600(800)$ for left(right) Figs.
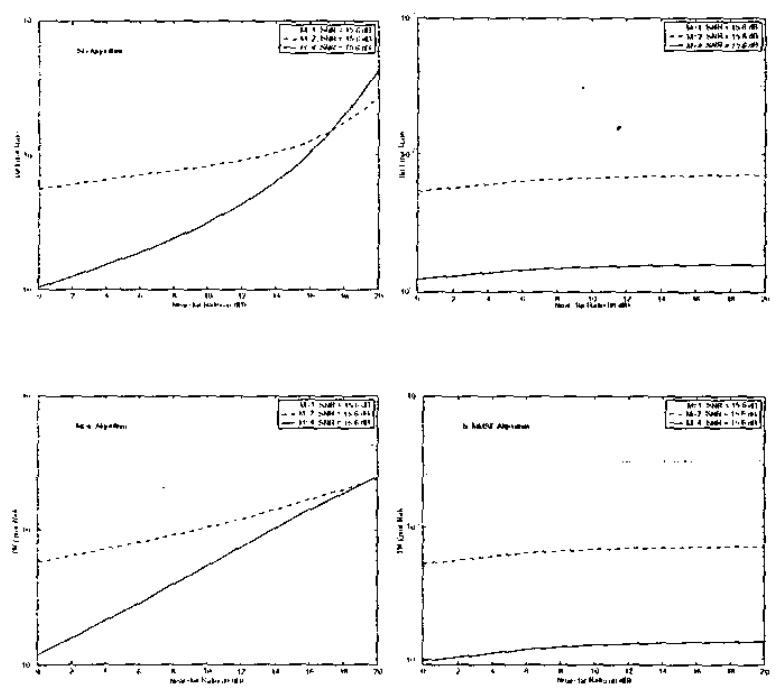

Fig. 3. BER performance of adaptive receivers as a function of NFR and number of transmit antennas. $\rho_{12}=-0.28, \rho_{21}=-0.48, \mathrm{SNR}=15.6 \mathrm{~dB}$

In Fig. 2, we compare the convergence behaviour of the algorithms in a dynamic multiuser environment where users may dynamically enter and exit the system. A dynamic multiuser environment is simulated wherein the near-far ratio is increased from 10 to $18 \mathrm{~dB}$ (i.e., the power of the interfering user is increased by $8 \mathrm{~dB}$, or, equivalently, a new interferer with $8 \mathrm{~dB}$ of power enters the system) at the $n=400^{\text {th }}$ block, and the near-far ratio is reduced back to $10 \mathrm{~dB}$ (i.e., the power of the interferer comes down to the original value, or. equivalently, the interferer with $8 \mathrm{~dB}$ of power leaves the system) at the $n=600^{\text {th }}$ block. From the left figure of Fig. 2, it is observed that the RLS algorithm is able to track such dynamic power imbalances in the channel at a faster speed (and also converge to a smaller MSE) compared to the $S G$ algorithm. In the right figure of Fig. 2, we present a similar comparison between the MOE and the S-MMSE algorithm (no training data algorithms). It is obscrved that the S-MMSE is able to track the dynamic variations in the MAl much better (converge faster and to a smaller MSE) than the MOE algorithm.
In Fig. 3, we illustrate the near-far resistance performance of the algorithms as a function of number of transmit antennas at an SNR of $15 \mathrm{~dB}$. The transmit power on each of the transmit antennas is scaled such that the total transmit power is the same in all the cases of $M=1,2,4$. We see that the $\mathrm{SG}$ algorithm, while being reasonably near-far resistant for the cases of one and two transmit antennas $(M=1,2)$, drastically looses its near-far resistance for four transmit antennas $(M=4)$. For example, at a NFR of $20 \mathrm{~dB}$, the performance with four transmit antennas degrades to that of one transmit antenna, thus nullifying the diversity benefit of multiple transmit antennas. A similar performance behaviour can be observed for the MOE algorithm. This performance degradation at larger number of transmit antennas can be attributed to the increase in the value of $Q(K-1)(Q \geq M)$, the number of interfering bits per block. On the other hand, the RLS and the S-MMSE algorithms maintain their nearfar resistance very well even when the number of transmit antennas is increased. Hence, clearly, RLS and S-MMSE algorithms are preferred in multituser, multiple transmit antenna schemes from a near-far resistance point-of-view. However, being less complex than other adaptive detectors the SG algorithm may still be a preferred. In such a case, some transmit power control can be employed to compensate for the near-far resistance loss when more number of transmit antennas are used.

\section{CONClusions}

We presented a comparative performance evaluation of adaptive multiuser detectors under near-far conditions in a space-time coded CDMA system. We showed that increasing in number of transmit antennas degraded the near-far resistance of the stochastic gradient (SG) and the minimum output energy (MOE) detectors significantly at high near-far ratios, to the extent that the diversity benefit of multiple transmit antennas is nullified. The recursive least squares (RLS) and the subspace based MMSE (S-MMSE) algorithms, on the other hand, were shown to maintain their near-far resistance without loosing much on the diversity gain, when the numbel of transmit antennas is increased.

\section{REFERENCES}

[1] V. Tarokh, H. Jafarkhani, and A. R. Calderbank, "Space-time blo" codes from orthogonal designs." IEEE Trans. Inform. Theory vol. 4 no. 5. pp. 1456-1467. July 1999.

[2] B. Hochwald. T. L. Marzetta, and C. B Papadias, "A transmiti diversity scheme for wideband CDMA systems based on space-i., spreading," IEEE JSAC, vol. 19, no. 1, pp. 48-60, January 2001.

[3] S. Verdu, Multiuser Detection, Cambridge University Press. 1998.

[4] X. Wang and H. V. Poor. "Space-ime multiuser defection in multip: CDMA channels," IEEE Trans. Sig. Proc., vol. 47, no. 9, Sept. 195

[5] H. Huang and H. Viswanathan, "Multiple antennas and muitit: detection in high data rate CDMA systems." Proc. IEEE VTC'200"

[6] X. Wang and $H$. V. Poor, "Blind mustiuser detection: A subs:approach." IEEE Trans: Inform. Theory. pp. 677-690, March 1998. 